PROCEEDINGS OF THE

AMERICAN MATHEMATICAL SOCIETY

Volume 140, Number 1, January 2012, Pages 357-366

S 0002-9939(2011)10893-1

Article electronically published on May 25, 2011

\title{
INFINITE SEQUENCES OF MUTUALLY NON-CONJUGATE SURFACE BRAIDS REPRESENTING SAME SURFACE-LINKS
}

\author{
MASAHIDE IWAKIRI \\ (Communicated by Daniel Ruberman)
}

\begin{abstract}
We give an infinite sequence of mutually non-conjugate surface braids with same degree representing the trivial surface-link with at least two components and a pair of non-conjugate surface braids with same degree representing a spun $(2, t)$-torus knot for $t \geq 3$. To give these examples, we introduce new invariants of conjugacy classes of surface braids via colorings by Alexander quandles or core quandles of groups.
\end{abstract}

\section{INTRODUCTION}

In 1-dimensional knot theory, links and braids are closely related by Alexander's theorem and Markov's theorem; i.e., any link type in $\mathbf{R}^{3}$ can be represented as the closure of a braid, and two braids represent the same link type if and only if they are related by conjugations, stabilizations and destabilizations. A stabilization or a destabilization changes the number of strands, although a conjugation keeps it. There is a natural question: Can two braids of $n$ strands representing the same link types be related by conjugations? The answer is negative. The first counterexample was given by H. R. Morton [11, who showed that there exists an infinite sequence of mutually non-conjugate braids of 4 strands representing the trivial knot (cf. [2]). This result was generalized in [3, 12, 13, 14, 15]. In this paper, we would like to study a similar problem for surface-links and surface braids.

In 2-dimensional knot theory, there are similar theorems to Alexander's theorem and Markov's theorem; i.e., any surface-link type in $\mathbf{R}^{4}$ is represented as the closure of a surface braid, and two surface braids represent the same surface-link type if and only if they are related by braid ambient isotopies, conjugations, stabilizations and destabilizations. Alexander's theorem in dimension four was announced by O. Viro [17 and proved by S. Kamada 7, and Markov's theorem in dimension four was proved by S. Kamada [8, 10. I. Hasegawa 4 gave the first examples of a pair of non-conjugate surface braids with degree 5 representing a 4-component surface-link. Here, degree is the number of 2-dimensional "strands". (See $\S 2$ for the concrete definition.) We prove the following theorems in this paper.

Theorem 1.1. There is an infinite sequence of mutually non-conjugate surface braids with degree $2 s$ representing the trivial s-component surface-link for any $s \geq 2$ and any genus.

Received by the editors July 16, 2010 and, in revised form, November 11, 2010 and November $12,2010$.

2010 Mathematics Subject Classification. Primary 57Q45.

(C)2011 American Mathematical Society Reverts to public domain 28 years from publication 
Theorem 1.2. There is a pair of non-conjugate surface braids with degree 4 representing a spun $(2, t)$-torus knot for $t \geq 3$.

To prove these theorems, we introduce new invariants of conjugacy classes of surface braids via colorings by Alexander quandles or core quandles of groups. These invariants are very simple and useful.

This paper consists of four sections. We review surface braids and their chart descriptions in $\S 2$. In $\S 3$, we define invariants of conjugacy classes of surface braids. In $\S 4$, we prove our theorems.

\section{Preliminaries}

A surface-link $F$ is a closed oriented surface embedded in Euclidean 4-space $\mathbf{R}^{4}$ locally flatly. If $F$ is connected, then it is called a surface-knot. A 2-link is a surface-link consisting of 2-spheres. A surface-knot $F$ is trivial if $F$ bounds a handlebody in $\mathbf{R}^{4}$, and a surface-link $F$ is trivial if $F$ is a split union of trivial surface-knots. Two surface-links $F$ and $F^{\prime}$ are equivalent if there is an orientationpreserving homeomorphism $f: \mathbf{R}^{4} \longrightarrow \mathbf{R}^{4}$ such that $f(F)=F^{\prime}$.

A surface braid $S$ of degree $m$ is an oriented surface embedded in $D_{1} \times D_{2}$ locally flatly and properly such that the restriction map $\left.\pi\right|_{S}$ of the projection map $\pi: D_{1} \times D_{2} \longrightarrow D_{2}$ is an $m$-fold branched covering map and $\partial S=X_{m} \times \partial D_{2}$, where $D_{1}$ and $D_{2}$ are 2-disks and $X_{m}$ is a fixed set of $m$ interior points of $D_{1}$. If the branched covering map is simple, then $S$ is called simple.

Two surface braids $S$ and $S^{\prime}$ with same degree are equivalent if they are ambient isotopic by a fiber-preserving isotopy $\left\{h_{u}\right\}_{0 \leq u \leq 1}$ of $D_{1} \times D_{2}$, as a $D_{1}$-bundle over $D_{2}$, rel $D_{1} \times \partial D_{2}$. Two surface braids $S$ and $S^{\prime}$ are braid ambient isotopic if they are ambient isotopic by an isotopy $\left\{h_{u}\right\}_{0 \leq u \leq 1}$ of $D_{1} \times D_{2}$ rel $D_{1} \times \partial D_{2}$ such that $h_{u}(S)$ is a surface braid and $\left.h_{u}\right|_{D_{1} \times \partial D_{2}}=i d$ for $\forall u \in[0,1]$. If two surface braids $S$ and $S^{\prime}$ are equivalent, then they are braid ambient isotopic. There is an example of a pair of surface braids that are braid ambient isotopic but not equivalent (cf. [10]).

For a surface braid $S$ of degree $m$, we have a surface-link obtained from $S$ by attaching $m$ parallel 2-disks onto the boundary of $S$ in $\overline{\mathbf{R}^{4} \backslash D_{1} \times D_{2}}$. We call the surface the closure of $S$.

S. Kamada introduced charts to represent surface braids. An $m$-chart $\Gamma$ is a (possibly empty) finite graph in an oriented 2-disk $D_{2}$, which may have hoops (that are closed edges without vertices), satisfying the following conditions:

(i) Every vertex has degree one, four or six.

(ii) Every edge is directed and labeled by an integer in $\{1,2, \ldots, m-1\}$.

(iii) For each vertex of degree six, three consecutive edges are directed inward and the other three are directed outward; these six edges are labeled by $i$ and $i+1$ alternately for some $i$.

(iv) For each vertex of degree four, two consecutive edges are directed inward and the other two are directed outward; these four edges are labeled by $i$ and $j$ alternately with $|i-j|>1$.

An example of a 4-chart is given in Figure 1. A vertex of degree one or six is called a black vertex or a white vertex, respectively. An edge attached to a white vertex is called a middle edge if it is the middle of the three consecutive edges which 


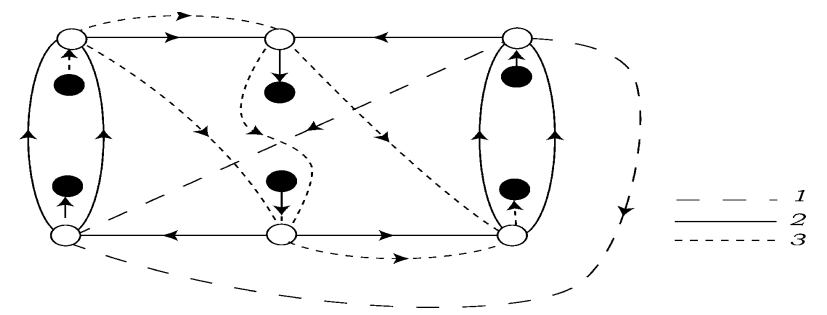

FiguRE 1. Example of a 4-chart

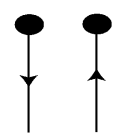

black vertices

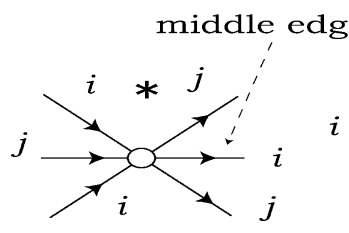

$|i-j|=1$ $i$

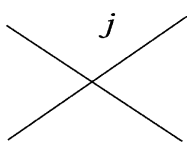

$|i-j|>1$

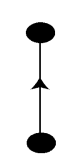

free edge

white vertex

Figure 2
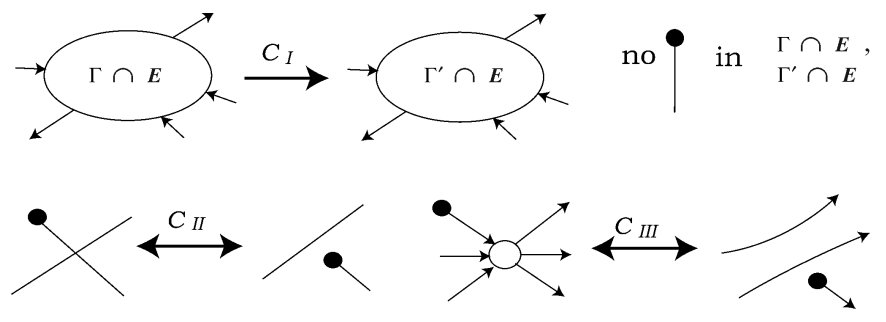

Figure 3. $C$-moves

are oriented in the same direction; otherwise it is called a non-middle edge. A free edge is an edge in a chart whose endpoints are black vertices. See Figure 2.

Operations listed below (and their inverses) are called a $C_{I^{-}}, C_{I I^{-}}$and $C_{I I I^{-}}$ move, respectively. See Figure 3 . These moves are called $C$-moves. Two $m$-charts are $C$-move equivalent if they are related by a finite sequence of such $C$-moves and ambient isotopies.

$\left(C_{I}\right)$ For a 2-disk $E$ on $D_{2}$ such that $\Gamma \cap E$ has no black vertices, replace $\Gamma \cap E$ with an arbitrary chart that has no black vertices.

$\left(C_{I I}\right)$ Suppose that there is an edge $\alpha$ attached to a black vertex $B$ and a 4 -valent vertex $v$. Remove $\alpha$ and $v$, attach $B$ to the diagonal edge of $\alpha$ and connect the other two edges in a natural way.

$\left(C_{I I I}\right)$ Let a black vertex $B$ and a white vertex $W$ be connected by a non-middle edge $\alpha$ of $W$. Remove $\alpha$ and $W$, attach $B$ to the edge of $W$ opposite to $\alpha$, and connect other four edges in a natural way.

In [10], S. Kamada proved that there is a one-to-one correspondence between equivalence classes of simple surface braids of degree $m$ and $C$-move equivalence 


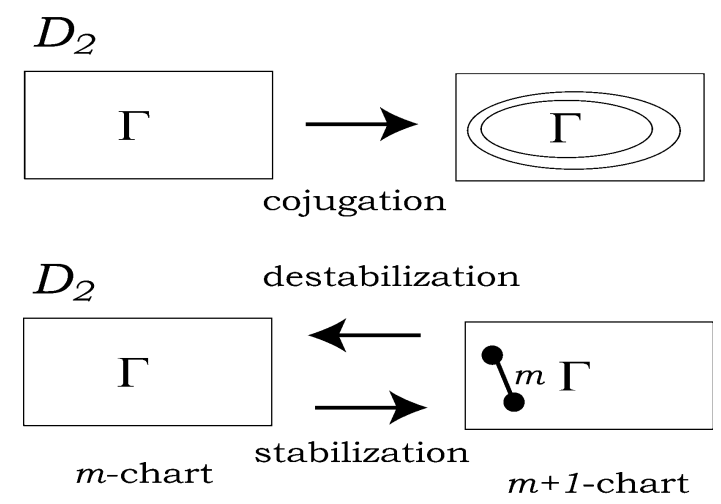

Figure 4. Conjugations, stabilizations and destabilizations

classes of $m$-charts. For a chart $\Gamma$, we denote by $S(\Gamma)$ the closure of a simple surface braid corresponding to $\Gamma$. We call $S(\Gamma)$ a surface-link represented by $\Gamma$.

A conjugation for a chart is an operation inserting some boundary parallel hoops. For an $m$-chart $\Gamma$, an $m+1$-chart is obtained from $\Gamma$ by inserting a free edge labeled by $m$. This operation is called a stabilization, and the inverse operation is called a destabilization. See Figure 4. A conjugation, stabilization and destabilization for a surface braid are operations corresponding to a conjugation, stabilization and destabilization for a chart, respectively. We have the following lemma.

Lemma 2.1 ([9, 10]). If $\Gamma$ and $\Gamma^{\prime}$ are related to each other by $C$-moves, conjugations, stabilizations and destabilizations, then $S(\Gamma)$ and $S\left(\Gamma^{\prime}\right)$ are equivalent.

\section{INVARIANTS}

In this section, we review quandle colorings of a chart [4, 6] and introduce invariants of conjugacy classes of surface braids.

A quandle is a set $X$ with a binary operation $*: X \times X \longrightarrow X$ satisfying the following properties:

(a) For any $x \in X, x * x=x$.

(b) For any $x_{1}, x_{2} \in X$, there is a unique $x_{3} \in X$ such that $x_{1}=x_{3} * x_{2}$.

(c) For any $x_{1}, x_{2}, x_{3} \in X,\left(x_{1} * x_{2}\right) * x_{3}=\left(x_{1} * x_{3}\right) *\left(x_{2} * x_{3}\right)$.

Example 3.1. (i) The set $\mathbf{Z}_{n}(\cong \mathbf{Z} / n \mathbf{Z})$ becomes a quandle under the binary operation $a * b=2 b-a(\bmod n)$, which is called the dihedral quandle $R_{n}$ of order $n$.

(ii) Set $\Lambda:=\mathbf{Z}\left[t, t^{-1}\right]$. A $\Lambda$-module $M$ becomes a quandle under the binary operation $a * b=t a+(1-t) b$, which is called an Alexander quandle. If $M=$ $\Lambda /(n, t+1)$, then $M$ is isomorphic to $R_{n}$.

(iii) A group $G$ becomes a quandle under the binary operation $a * b=b a^{-1} b$, which is called the core quandle of $G$. The core quandle of $\mathbf{Z}_{n}$ is isomorphic to $R_{n}$.

Let $\Gamma$ be an $m$-chart, and the set of regions of $D_{2} \backslash \Gamma$ be denoted by $\Sigma(\Gamma)$. A map $C: \Sigma(\Gamma) \longrightarrow X^{m}$ is an $X$-coloring of $\Gamma$ if it is such that $C\left(\lambda_{1}\right)=\left(y_{1}, \ldots, y_{m}\right)$ and $C\left(\lambda_{2}\right)=\left(y_{1}, \ldots, y_{i-1}, y_{i+1}, y_{i} * y_{i+1}, y_{i+2}, \cdots, y_{m}\right)$ for each edge $e$ with label $i$, where $\lambda_{1}$ and $\lambda_{2}$ are regions separated by $e$ and $\lambda_{1}$ is on the left side of $e$. See 


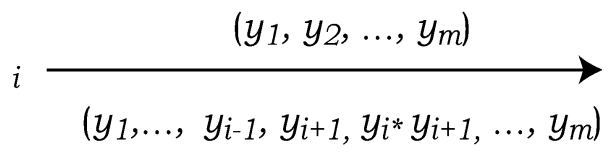

FiguRE 5. Coloring condition

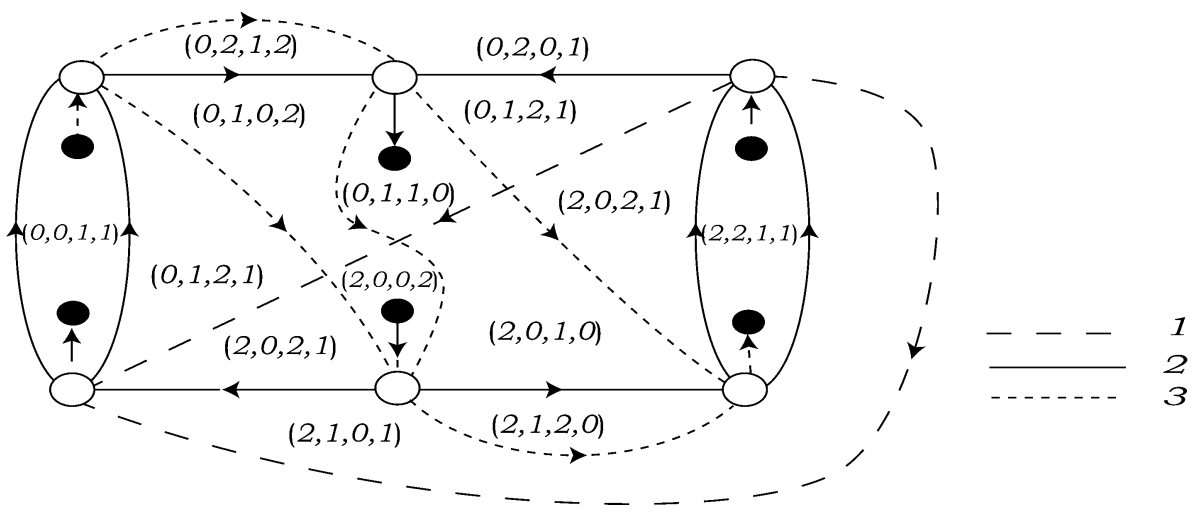

FiguRE 6. Example of an $R_{3}$-coloring

Figure 5. The set of $X$-colorings of $\Gamma$ is denoted by $\operatorname{Col}_{X}(\Gamma)$. An example of an $R_{3}$-coloring of a 4-chart is given in Figure 6. If $C(\lambda)=(y, \ldots, y)$ for $\lambda \in \Sigma(\Gamma)$ and for some $y \in X$, then we call $C$ by a trivial $X$-coloring.

Remark 3.2. The above definition of a quandle coloring of a chart is a natural interpretation of a quandle coloring of a surface-link represented by the chart. It is known that the number of quandle colorings are invariants of surface-links. Furthermore, it is also known that if two $m$-charts $\Gamma$ and $\Gamma^{\prime}$ are related by a $C$-move or a conjugation, then there is one-to-one correspondence between $\operatorname{Col}_{X}(\Gamma)$ and $\operatorname{Col}_{X}\left(\Gamma^{\prime}\right)$ for any quandle $X$. This can be proved only in terms of charts by checking for a conjugation, a $C_{I I}$-move, a $C_{I I I}$-move or generators of $C_{I}$-moves given in [1, 16. It is noted that the one-to-one equivalence does not change colors of regions that are fixed by a $C$-move or a conjugation for any $X$-coloring.

Let $\Gamma$ be an $m$-chart and $X$ be an Alexander quandle or the core quandle of a group. We define a map $\kappa: \operatorname{Col}_{X}(\Gamma) \times \Sigma(\Gamma) \longrightarrow X$ by

$$
\kappa(C, \lambda)=\sum_{i=1}^{m} t^{m-i} y_{i}
$$

when $X$ is an Alexander quandle and by

$$
\kappa(C, \lambda)=\prod_{i=1}^{m} y_{i}^{(-1)^{m-i}}
$$


when $X$ is the core quandle of a group, where $C(\lambda)=\left(y_{1}, y_{2}, \ldots, y_{m}\right)$ for $\lambda \in \Sigma(\Gamma)$. Suppose that $X=R_{n}$. Then by Equation (11),

$$
\kappa(C, \lambda)=\sum_{i=1}^{m} t^{m-i} y_{i}=\sum_{i=1}^{m}(-1)^{m-i} y_{i} .
$$

On the other hand, by Equation (2),

$$
\kappa(C, \lambda)=\prod_{i=1}^{m} y_{i}^{(-1)^{m-i}}=\sum_{i=1}^{m}(-1)^{m-i} y_{i} .
$$

Thus, the two definitions in Equations (11) and (2) coincide. If $X$ is an Alexander quandle and the core quandle of a group, then $X$ is a dihedral quandle. Thus, $\kappa(C, \lambda)$ is well-defined.

Lemma 3.3. The map $\kappa(C, \lambda)$ is independent of the choice of $\lambda$.

Proof. Let $\lambda_{1}$ (or $\lambda_{2}$ ) be a region that is the left side (or right side) of an edge labeled by $i \in\{1, \ldots, m-1\}$. It is sufficient to prove that $\kappa\left(C, \lambda_{1}\right)=\kappa\left(C, \lambda_{2}\right)$ for an $X$ coloring $C$. Set $C\left(\lambda_{1}\right)=\left(y_{1}, y_{2}, \ldots, y_{m}\right)$. Then $C\left(\lambda_{2}\right)=\left(y_{1}, \ldots, y_{i-1}, y_{i+1}, y_{i} *\right.$ $\left.y_{i+1}, y_{i+2}, \ldots, y_{m}\right)$. If $X$ is an Alexander quandle, then

$$
\begin{aligned}
\kappa\left(C, \lambda_{1}\right)-\kappa\left(C, \lambda_{2}\right) & =\left(t^{m-i} y_{i}+t^{m-i-1} y_{i+1}\right)-\left(t^{m-i} y_{i+1}+t^{m-i-1}\left(y_{i} * y_{i+1}\right)\right) \\
& =\left(t^{m-i}-t^{m-i-1} t\right) y_{i}+\left(t^{m-i-1}-t^{m-i}-t^{m-i-1}(1-t)\right) y_{i+1} \\
& =0 .
\end{aligned}
$$

If $X$ is the core quandle of a group, then we have

$$
x y^{-1}=y y^{-1} x y^{-1}=y(x * y)^{-1},
$$

for any $x, y \in X$. Thus,

$$
\begin{aligned}
\kappa\left(C, \lambda_{1}\right) & =\prod_{i=1}^{m} y_{i}^{(-1)^{m-i}} \\
& =\left(\prod_{i=1}^{j-1} y_{i}^{(-1)^{m-i}}\right) y_{j}^{(-1)^{m-j}} y_{j+1}^{(-1)^{m-j-1}}\left(\prod_{i=j+2}^{m} y_{i}^{(-1)^{m-i}}\right) \\
& =\left(\prod_{i=1}^{j-1} y_{i}^{(-1)^{m-i}}\right) y_{j+1}^{(-1)^{m-j}}\left(y_{j} * y_{j+1}\right)^{(-1)^{m-j-1}}\left(\prod_{i=j+2}^{m} y_{i}^{(-1)^{m-i}}\right) \\
& =\kappa\left(C, \lambda_{2}\right) .
\end{aligned}
$$

Therefore we have $\kappa\left(C, \lambda_{1}\right)=\kappa\left(C, \lambda_{2}\right)$.

By Lemma 3.3. we denote $\kappa(C, \lambda)$ by $\kappa(C)$. We define a multi-set

$$
K_{X}(\Gamma):=\left\{\kappa(C) \mid C \in \operatorname{Col}_{X}(\Gamma)\right\} .
$$

Theorem 3.4. A multi-set $K_{X}(\Gamma)$ is an invariant under $C$-moves and conjugations of charts, and hence $K_{X}(\Gamma)$ is also an invariant of conjugacy classes of surface braids.

Proof. Let $\Gamma$ and $\Gamma^{\prime}$ be $m$-charts such that $\Gamma^{\prime}$ is obtained from $\Gamma$ by a $C$-move or a conjugation. By Remark 3.2, there is one-to-one equivalence between $\operatorname{Col}_{X}(\Gamma)$ and $\operatorname{Col}_{X}\left(\Gamma^{\prime}\right)$. Let $C$ be an $X$-coloring of $\Gamma$ and $C^{\prime}$ be the $X$-coloring of $\Gamma^{\prime}$ corresponding to $C$. By Remark 3.2 and Lemma 3.3, $\kappa(C)=\kappa\left(C^{\prime}\right)$. Thus, we prove this theorem. 


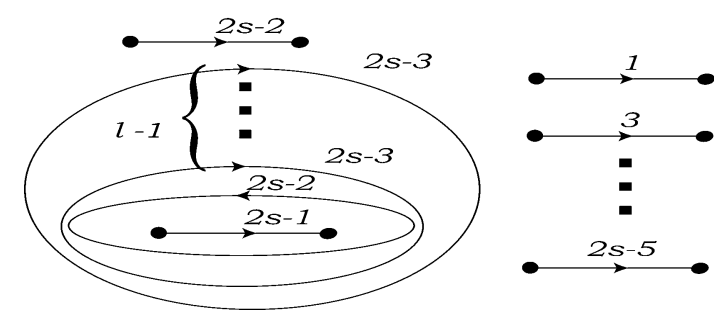

FiguRE $7 . A_{s}^{l}$

An oval nest is a free edge together with some concentric hoops. A chart is ribbon if it is $C$-move equivalent to a chart consisting of some oval nests.

Remark 3.5. In 4, I. Hasegawa defined another invariant of conjugacy classes of surface braids. Hasegawa's invariant requires that any surface braid corresponding to a ribbon chart has a specific value. By Hasegawa's invariant, we have the first example of a non-ribbon chart representing a ribbon surface-link and a pair of nonconjugate surface braids. The invariants $K_{X}$ do not help us to study whether a chart is ribbon or not, but they are useful in studying whether two ribbon charts are conjugate or not, as in $\S 4$.

\section{Proofs}

Let $A_{s}^{l}$ be a $2 s$-chart depicted in Figure 7 for natural numbers $s$ and $l$ with $s \geq 2$.

Lemma 4.1. A 2s-chart $A_{s}^{l}$ represents an s-component trivial 2-link.

Proof. By a destabilization and $C_{I}$-moves, $A_{s}^{l}$ can be deformed into a $2 s-1$-chart consisting of free edges labeled by $1,3, \ldots, 2 s-5$ and $2 s-2$. This chart represents an $s$-component trivial 2-link.

Let $\lambda$ be the region of $\Sigma\left(A_{s}^{l}\right)$ with $\partial \lambda \supset \partial D_{2}$ and $\lambda^{\prime}$ be the region with a free edge labeled by $2 s-1$ in $\partial \lambda$. We define integers $j_{1}, \ldots, j_{s}$ by $j_{i}=2 i-1$ for $1 \leq i \leq s-2$ and $i=s, j_{s-1}=2 s-2$. For non-negative integers $g_{1}, \ldots, g_{s}$, let $B_{s, g_{1}, \ldots, g_{s}}^{l}$ be a $2 s$-chart obtained from $A_{s}^{l}$ by inserting $g_{i}$ free edges in $\lambda$ labeled by $j_{i}$ for every $1 \leq i \leq s-1$ and $g_{s}$ free edges in $\lambda^{\prime}$ labeled by $j_{s}$. Then $B_{s, 0, \ldots, 0}^{l}=A_{s}^{l}$.

Lemma 4.2. A $2 s$-chart $B_{s, g_{1}, \ldots, g_{s}}^{l}$ represents an s-component trivial surface-link whose components have genera $g_{1}, \ldots, g_{s}$.

Proof. A free edge for a chart $\Gamma$ corresponds to a 1-handle attached to $S(\Gamma)$. Let $h_{i}$ be a 1 -handle for the free edge labeled by $j_{i}$ in $A_{s}^{l}$ for any $1 \leq i \leq s$. Insertion of a free edge labeled by $j_{i}$ for some $1 \leq i \leq s$ means the surgery along a 1-handle $h$ parallel to $h_{i}$. Sliding $h$ along $h_{i}$ for some $i \in\{1, \ldots, s\}$, we see that $h$ is a trivial 1-handle in the sense of $\left[5\right.$. Since $A_{s}^{l}$ represents an $s$-component trivial 2-link by Lemma 4.1, the resultant surface-link is also trivial. Repeating this, we see that $B_{s, g_{1}, \ldots, g_{s}}^{l}$ represents an $s$-component trivial surface-link whose components have genera $g_{1}, \ldots, g_{s}$. 


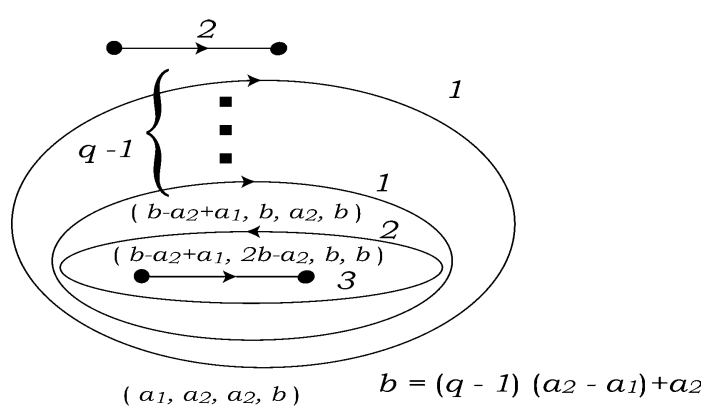

FIGURE 8

Lemma 4.3. Let $p, q$ be odd prime integers. Then

$$
K_{R_{p}}\left(B_{s, g_{1}, \ldots, g_{s}}^{q}\right)= \begin{cases}\{\underbrace{0, \ldots, 0}_{p^{s}}\}, & p=q, \\ \{\underbrace{0, \ldots, 0}_{p^{s-1}}, \underbrace{1, \ldots, 1}_{p^{s-1}}, \ldots, \underbrace{p-1, \ldots, p-1}_{p^{s-1}}\}, & p \neq q .\end{cases}
$$

Proof. By Lemma 4.2, there are $p^{s} R_{p}$-colorings of $B_{s, g_{1}, \ldots, g_{s}}^{q}$. Let $\lambda$ be the region with $\partial \lambda \supset \partial D_{2}$. We see that $\operatorname{Col}_{R_{p}}\left(B_{s, g_{1}, \ldots, g_{s}}^{q}\right)=\left\{C_{a_{1}, a_{2}, \ldots, a_{s}}\right\}_{a_{1}, a_{2}, \ldots, a_{s} \in R_{p}}$, where $C_{a_{1}, a_{2}, \ldots, a_{s}}$ is an $R_{p}$-coloring such that

$C_{a_{1}, a_{2}, \ldots, a_{s}}(\lambda)=\left(a_{1}, a_{1}, a_{2}, a_{2}, \ldots, a_{s-2}, a_{s-2}, a_{s-1}, a_{s}, a_{s},(q-1)\left(a_{s}-a_{s-1}\right)+a_{s}\right)$

for any $a_{1}, a_{2}, \ldots, a_{s} \in R_{p}$. See Figure 8 for $B_{2,0,0}^{q}$. Then

$$
\begin{aligned}
\kappa\left(C_{a_{1}, a_{2}, \ldots, a_{s}}\right) & \left.=-a_{1}+a_{1}-\cdots-a_{s-1}+a_{s}-a_{s}+(q-1)\left(a_{s}-a_{s-1}\right)+a_{s}\right) \\
& =q\left(a_{s}-a_{s-1}\right) .
\end{aligned}
$$

Since both $p$ and $q$ are prime, we prove this lemma.

Proof of Theorem 1.1. By Theorem 3.4 and Lemmas 4.2 and 4.3, we have Theorem 1.1 .

If an $m$-chart $\Gamma$ cannot be deformed into an $m$-1-chart $\Gamma^{\prime}$ with a free edge labeled by $m-1$ by $C$-moves and conjugations, then we say that $\Gamma$ is weakly irreducible. If an $m$-chart $\Gamma$ cannot be deformed into an $m-1$-chart $\Gamma^{\prime}$ with a free edge labeled by $m-1$ by $C$-moves, conjugations and braid ambient isotopies, then we say that $\Gamma$ is strongly irreducible.

Remark 4.4. Suppose that $g_{1}, \ldots, g_{s}$ are positive integers. Then the braid index of a surface-link represented by $B_{s, g_{1}, \ldots, g_{s}}^{q}$ is at least $2 s$, and hence we cannot apply a destabilization to $B_{s, g_{1}, \ldots, g_{s}}^{q}$. Thus, $B_{s, g_{1}, \ldots, g_{s}}^{q}$ is strongly irreducible. We do not know the difference between strong and weak irreducibility.

Proof of Theorem 1.2, It is known that a spun $(2, t)$-torus knot is represented by a 3 -chart $C^{t}$ depicted in Figure 9 ([10]). Let $D^{t}$ and $E^{t}$ be 4 -charts depicted in Figure 10 for odd $t$. By a similar argument to the proof of Lemma 4.1, both $D^{t}$ and $E^{t}$ can be deformed into $C^{t}$ by a destabilization and $C_{I}$-moves. Let $\lambda_{D}\left(\right.$ or $\left.\lambda_{E}\right)$ be the region with $\partial \lambda_{D}\left(\right.$ or $\left.\partial \lambda_{E}\right) \supset \partial D_{2}$. We see that $\operatorname{Col}_{R_{t}}\left(D^{t}\right)=\left\{C_{a, b}^{D}\right\}_{a, b \in R_{t}}$ 


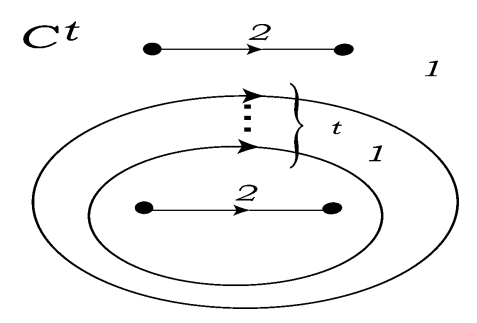

Figure 9. $C^{t}$

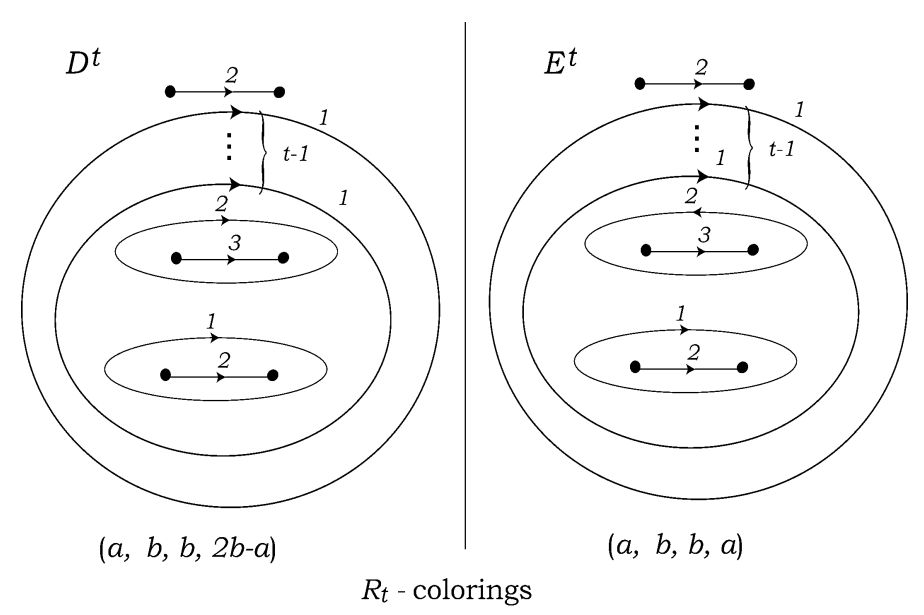

Figure 10. $D^{t} \mid E^{t}$

and $\operatorname{Col}_{R_{t}}\left(E^{t}\right)=\left\{C_{a, b}^{E}\right\}_{a, b \in R_{t}}$, where $C_{a, b}^{D}\left(\right.$ or $\left.C_{a, b}^{E}\right)$ is an $R_{t}$-coloring of $D^{t}$ (or $E^{t}$ ) such that

$$
\begin{gathered}
C_{a, b}^{D}\left(\lambda_{D}\right)=(a, b, b, 2 b-a), \\
C_{a, b}^{D}\left(\lambda_{E}\right)=(a, b, b, a)
\end{gathered}
$$

for any $a, b \in R_{t}$. Thus, we have

$$
\begin{gathered}
K_{R_{t}}\left(D^{t}\right)=\{\underbrace{0, \ldots, 0}_{t}, \underbrace{1, \ldots, 1}_{t}, \ldots, \underbrace{t-1, \ldots, t-1}_{t}\}, \\
K_{R_{t}}\left(E^{t}\right)=\{\underbrace{0, \ldots, 0}_{t^{2}}\} .
\end{gathered}
$$

By Theorem 3.4, $D^{t}$ and $E^{t}$ are non-conjugate.

\section{ACKNOWLEDGMENTS}

The author would like to thank Kengo Kishimoto, Reiko Shinjo, Kokoro Tanaka and Shin Satoh for helpful comments. 


\section{REFERENCES}

1. J. S. Carter and M. Saito, Braids and movies, J. Knot Theory Ramifications 5 (1996), 589608. MR1414089 (97j:57028)

2. T. Fiedler, A small state sum for knots, Topology 32 (1993), 281-294. MR.1217069 (94c:57006)

3. E. Fukunaga, An infinite sequence of conjugacy classes in the 4-braid group representing a torus link of type $(2, k)$, preprint.

4. I. Hasegawa, A certain linear representation of the classical braid group and its application to surface braids, Math. Proc. Cambridge Philos. Soc. 141 (2006), 287-301. MR.2265876 (2008e:57017)

5. F. Hosokawa and A. Kawauchi, Proposals for unknotted surfaces in four-spaces, Osaka J. Math. 16 (1979), 233-248. MR.527028 (81c:57018)

6. M. Iwakiri, The lower bound of the w-indices of surface links via quandle cocycle invariants, Trans. Amer. Math. Soc. 362 (2010), 1189-1210. MR2563726 (2010j:57033)

7. S. Kamada, A characterization of groups of closed orientable surfaces in 4-space, Topology 33 (1994), 113-122. MR1259518 (95a:57002)

8. S. Kamada, Alexander's and Markov's theorems in dimension four, Bull. Amer. Math. Soc. (N.S.) 31 (1994), 64-67. MR.1254074 (94j:57023)

9. S. Kamada, An observation of surface braids via chart description, J. Knot Theory Ramifications 4 (1996), 517-529. MR1406718 (97j:57009)

10. S. Kamada, Braid and knot theory in dimension four, Math. Surveys Monogr. 95, Amer. Math. Soc., 2002. MR1900979 (2003d:57050)

11. H. R. Morton, An irreducible 4-string braid with unknotted closure, Math. Proc. Cambridge Philos. Soc. 93 (1983), 259-261. MR691995 (84m:57006)

12. R. Shinjo, An infinite sequence of non-conjugate 4-braids representing the same knot of braid index 4, Intelligence of low dimensional topology 2006, 293-297, Ser. Knots Everything, 40, World Sci. Publ., 2007. MR2371738 (2008m:57033)

13. R. Shinjo, Non-conjugate braids whose closures result in the same knot, J. Knot Theory Ramifications 19 (2010), 117-124. MR2640995

14. A. Stoimenow, Lie groups, Burau representation, and non-conjugate braids with the same closure link, preprint.

15. A. Stoimenow, The density of Lawrence-Krammer and non-conjugate braid representations of links, preprint arXiv:0809.0033.

16. K. Tanaka, A note on CI-moves, Intelligence of low dimensional topology 2006, 307-314, Ser. Knots Everything, 40, World Sci. Publ., 2007. MR2371740 (2009a:57017)

17. O. Ya. Viro, Lecture given at Osaka City University, September, 1990.

Graduate School of Science, Osaka City University, 3-3-138 Sugimoto Sumiyoshi-ku, OSAKA 558-8585, JAPAN

E-mail address: iwakiri@sci.osaka-cu.ac.jp

Current address: Graduate School of Science and Engineering, Saga University, 1 Honjo-machi, Saga City, Saga, 840-8502, Japan

E-mail address: iwakiri@ms.saga-u.ac.jp 